To appear in the Astrophysical Journal

\title{
On the Detectability of Oxygen X-ray Fluorescence and its Use as a Solar Photospheric Abundance Diagnostic
}

\author{
Jeremy J. Drake ${ }^{1}$ \\ Barbara Ercolano ${ }^{1}$ \\ ${ }^{1}$ Smithsonian Astrophysical Observatory, MS-3, \\ 60 Garden Street, \\ Cambridge, MA 02138 \\ jdrake@cfa.harvard.edu
}

\begin{abstract}
Monte Carlo calculations of the $\mathrm{O} \mathrm{K} \alpha$ line fluoresced by coronal X-rays and emitted just above the temperature minimum region of the solar atmosphere have been employed to investigate the use of this feature as an abundance diagnostic. While quite weak, we estimate line equivalent widths in the range 0.02-0.2 $\AA$, depending on the X-ray plasma temperature. The line remains essentially uncontaminated by blends for coronal temperatures $T \leq 3 \times 10^{6} \mathrm{~K}$ and should be quite observable, with a flux $\gtrsim 2 \mathrm{ph} \mathrm{s}^{-1} \operatorname{arcmin}^{-2}$. Model calculations for solar chemical mixtures with an $\mathrm{O}$ abundance adjusted up and down by a factor of 2 indicate $35-60 \%$ changes in $\mathrm{O} \mathrm{K} \alpha$ line equivalent width, providing a potentially useful $\mathrm{O}$ abundance diagnostic. Sensitivity of equivalent width to differences between recently recommended chemical compositions with "high" and "low" complements of the CNO trio important for interpreting helioseismological observations is less accute, amounting to $20-26 \%$ at coronal temperatures $T \leq 2 \times 10^{6} \mathrm{~K}$. While still feasible for discriminating between these two mixtures, uncertainties in measured line equivalent widths and in the models used for interpretation would need to be significantly less than $20 \%$. Provided a sensitive X-ray spectrometer with resolving power $\geq 1000$ and suitably well-behaved instrumental profile can be built, X-ray fluorescence presents a viable means for resolving the solar "oxygen crisis".
\end{abstract}

Subject headings: Sun: abundances — Sun: activity — Sun: corona — Sun: X-rays - X-rays: stars - radiation mechanisms: nonthermal 


\section{Introduction}

At the turn of the millennium, precise agreement between the observed oscillation spectrum of the Sun and predictions of theoretical solar models, built using the best abundance assessments of the day (Grevesse \& Sauval 1998), represented a triumph of modern astrophysics (e.g. Bahcall et al. 2005a). This illustrious accord has recently been clouded by sophisticated re-assessments of the solar complement of light elements based on 3-D nonLTE hydrodynamic photospheric modelling: such models demand less of the elements C, $\mathrm{N}, \mathrm{O}$ and Ne that are important for the opacity of the solar interior by $25-35 \%$ compared to earlier assessments (Asplund et al. 2005). Solar models employing this chemical composition lead to predictions of the depth of the convection zone, helium abundance, density and sound speed in serious disagreement with helioseismology measurements (Basu \& Antia 2004; Turck-Chièze et al. 2004; Bahcall et al. 2005a). This state of affairs is now commonly referred to as the "solar model problem", or the "solar oxygen crisis" (e.g. Ayres et al. 2006).

To solve this problem, Antia \& Basu (2005) and Bahcall et al. (2005b) suggested the uncertain solar Ne abundance might be raised to compensate for lower $\mathrm{C}, \mathrm{N}$ and $\mathrm{O}$ abundances. Drake \& Testa (2005) found empirical support for this in Chandra high resolution X-ray spectra of mostly magnetically active stars for which the Ne/O abundance ratio appears consistently higher by a factor of $\sim 2$ or more than the currently recommended solar value of $\mathrm{Ne} / \mathrm{O}=0.15$ by number. They argued that existing solar coronal Ne/O measurements supporting the latter (see, e.g., Drake \& Testa 2005; Schmelz et al. 2005; Landi et al. 2007) might be biased by chemical fractionation. However, subsequent studies of the helioseismology data using different theoretical models disagree as to the viability of such a solution (Delahaye \& Pinsonneault 2006; Zaatri et al. 2007; Lin et al. 2007).

An alternative possibility is that the suggested downward $\mathrm{C}, \mathrm{N}$ and $\mathrm{O}$ abundance revisions are unwarranted. Avres et al. (2006) favour the Grevesse \& Sauval (1998) value of $\mathrm{O} / \mathrm{H}=8.83$ based on multi-component atmospheric modelling and analysis of CO lines. However, a very recent 3D empirical study of spatially-resolved spectropolarimetric observation of Fe I and O I lines by Socas-Navarro \& Norton (2007) finds $\mathrm{O} / \mathrm{H}=8.63 \pm 0.1$, in good agreement with Asplund et al. (2005).

The difficulty in determining the solar $\mathrm{O}$ abundance lies in the complexity of the nonlocal thermodynamic equilibrium optically-thick line formation problem in the inhomogeneous and turbulent photosphere. In this paper, we study a possible alternative approach. Drake \& Ercolano (2007) recently investigated the utility of the weak photospheric K $\alpha$ fluorescence line of neutral Ne excited by coronal X-ray photoionisation as an independent abundance diagnostic. Here, we turn our attention to the $\mathrm{O} \mathrm{K} \alpha$ line at $23.37 \AA(530.6 \mathrm{keV})$. 
In contrast to the complex photospheric absorption line formation problem, the formation of the X-ray fluorescent $\mathrm{O} \mathrm{K} \alpha$ line is relatively simple, depending only on the geometry of the overlying coronal source of irradiation and the heliocentric angle of the photospheric region in question (e.g. Bai 1979; Drake et al. 2007; Drake \& Ercolano 2007). We present Monte Carlo calculations of the fluorescent $\mathrm{O} \mathrm{K} \alpha$ line in $\S 2$, and address its potential as an abundance diagnostic in $\S 3$ and $\S 4$.

\section{Monte Carlo Calculations of Oxygen Fluorescence}

We adopt the same computational methods described in earlier work on $\mathrm{Fe}$ and $\mathrm{Ne}$ fluorescence lines, and the reader is referred to Drake et al. (2007, hereafter Paper I) and Drake \& Ercolano (2007, Paper II) for details. A brief summary is provided below.

X-ray "characteristic" (fluorescence) K $\alpha$ lines correspond to the $2 p-1 s$ decay of the excited state resulting from ejection of an inner-shell $1 s$ electron in the neutral (or nearneutral) atom by either electron impact or photoionisation. In the case of solar and stellar photospheric fluorescent lines, the former is expected to dominate (Basko 1978; Bai 1979; Parmar et al. 1984). For a given coronal X-ray source spectrum, $F(\lambda)$, the observed flux of $\mathrm{K} \alpha$ photons from the photosphere then depends on the photospheric abundance $A$ of the fluorescing species relative to that of other elements of significance for the photoabsorption opacity in the vicinity of the $1 s$ ionisation edge; the height $h$ of the emitting source; and the heliocentric angle $\theta$ between the emitting source and the observer (Bai 1979).

Fluorescent lines are formed in the region of the atmosphere corresponding to optical depth of approximately unity for the primary K-shell ionising photons. In the case of $\mathrm{O}$, for normal incidence the solar atmospheric Model C (VALC) of Vernazza, Avrett, \& Loeser (1981) reaches the K-shell photoabsorption $\tau=1$ depth slightly above the temperature minimum, at a gas temperature of about $5600 \mathrm{~K}$ and about $800 \mathrm{~km}$ above the point where the continuum optical depth at $5000 \AA$, $\tau_{5000}$, is unity.

The expected intensity of the emergent $\mathrm{O} \mathrm{K} \alpha$ line was computed using a modified version of the 3D Monte Carlo radiative transfer code MOCASSIN (Ercolano et al. 2003, 2005, see also Paper I for a specific description of fluorescence calculations). This code follows energy packets representing incident X-ray photons as they interact with the photospheric gas through photoelectric absorption or scattering events until the packets escape. O K $\alpha$ packets are produced following K-shell X-ray absorption with a probability dictated by the $\mathrm{K} \alpha$ fluorescent yield for oxygen: these packets are followed in the same fashion.

We adopted a value of $8.3 \times 10^{-3}$ for the fluorescence yield of neutral oxygen Krause 
1979). Coronal spectra used for the incident X-ray radiation field were computed using emissivities from the CHIANTI database (Landi et al. 2006) and the ion populations of Mazzotta et al. (1998), as implemented in the PINTofALE1 IDL suite of programs (Kashyap \& Drake 2000).

For a given coronal X-ray spectrum, the maximum intensity of a fluorescent line is achieved for a heliocentric angle $\theta=0$ and height $h=0$. As in the case of the earlier study of $\mathrm{Ne}$, since we are primarily interested here in the observability of the fluorescent line, we adopt $\theta=0$ and $h=0$ as baseline parameters, these values yielding the maximum possible line strength.

The wavelength of the $\mathrm{O} \mathrm{K} \alpha$ doublet has not been determined with high precision and we adopted the value $23.37 \pm 0.02 \AA$, corresponding to the mean of values found for the energy level of the excited $[1 s] 2 p^{4}{ }^{4} \mathrm{P}$ of $530.6 \pm 0.3 \mathrm{eV}$ from laboratory measurements by Krause (1994), Caldwell et al. (1994) and Stolte et al. (1997, see also the review by García et al. 2005). This wavelength is in good agreement with the position of the $2 p^{3}{ }^{4} S \rightarrow[1 s] 2 p^{4}{ }^{4} P$ resonance seen in absorption in the interstellar medium toward bright X-ray continuum sources by Juett et al. (2004).

The $\mathrm{O} \mathrm{K} \alpha$ flux was computed for isothermal irradiating coronal spectra with plasma temperatures in the range $10^{6}-10^{7} \mathrm{~K}$ and coronal and photospheric chemical compositions of Grevesse \& Sauval (1998, hereafter GS; with $\mathrm{O} / \mathrm{H}=8.83$ on the usual $\log +12$ scale) and Asplund et al. (2005, $\mathrm{O} / \mathrm{H}=8.66)$. As noted in $\S 1$, these two chemical mixtures differ not only in $\mathrm{O}$ abundance but also, primarily, in $\mathrm{C}, \mathrm{N}$ and $\mathrm{Ne}$ abundances: these differences turn out to be important for the $\mathrm{O}$ fluorescent line and are discussed in more detail below. To investigate changes in the $\mathrm{O}$ abundance only, we also performed calculations using a photospheric composition corresponding to the GS abundance mixture with $\mathrm{O}$ elevated and decreased by a factor of $2(\mathrm{O} / \mathrm{H}=9.13,8.53$, or $[\mathrm{O} / \mathrm{H}]= \pm 0.3)$.

\section{Strength of the $\mathrm{O} \mathbf{K} \alpha$ Line}

\subsection{Monte Carlo Results}

The photospheric $\mathrm{O} \mathrm{K} \alpha$ fluorescent line is shown in comparison with the direct coronal spectrum for the case of GS abundances for both coronal spectrum and photosphere in

\footnotetext{
${ }^{1}$ The Package for INTeractive Analysis of Line Emission is freely available from http://hea-www.harvard.edu/PINTofALE/
} 
Figure 1 for the range of coronal plasma temperatures $6.0 \leq \log T \leq 7.2$. The lower panel illustrates the same spectra seen at a resolving power of $\lambda / \Delta \lambda=1000$, where $\Delta \lambda$ is assumed to be the full-width at half-maximum of a Gaussian instrument response function. Unlike the $\mathrm{Ne} \mathrm{K} \alpha$ line, the $\mathrm{O} \mathrm{K} \alpha$ transition forms in a relatively uncrowded spectral region and the CHIANTI database predicts no devastatingly large blends in the immediate vicinity of $23.37 \AA$. For temperatures approaching $10^{7} \mathrm{~K}$, a line of Fe XXIII at $23.36 \AA$ appears, but for GS abundances is always dominated by the $\mathrm{O} K \alpha$ line by a factor of 10. Lying redward of our adopted line centre is $\mathrm{Ca}$ XV $\lambda 23.39$, which peaks at slightly cooler temperatures $(\log T \sim 6.6)$ at an intensity of about $30 \%$ of that of $\mathrm{O} \mathrm{K} \alpha$.

The full list of lines in the CHIANTI database within $5 \sigma$ of the $\mathrm{O} \mathrm{K} \alpha$ wavelength range $( \pm 0.05 \AA$ from $23.37 \pm 0.02 \AA)$ with intensities $\geq 10^{-4}$ times that of the brightest line (Fe XXIII $\lambda 23.363$ ) are listed in Table 4, The NIST Atomic Spectra Database (version 3.1.2; Ralchenko et al. 2007) also lists two other transitions within the $5 \sigma$ range from Ti XIII and Sc XIV. Neither of these are expected to be of any significance since their solar abundances according to the GS assessment are 300 and 21000 lower than that of Fe, respectively.

The equivalent width (EW) of the $\mathrm{O} \mathrm{K} \alpha$ line relative to coronal fluorescing spectra with GS abundances is illustrated in Figure 2 for a range of isothermal plasma temperatures and different photospheric $\mathrm{O}$ abundances. The Monte Carlo sampling error on the computed EWs is estimated to be no larger than $7 \%$. One striking feature of the trend of EW with $T$ for all compositions is the sharp decline with rising temperature for $\log T \lesssim 6.6$ : this contrasts with both $\mathrm{Ne} \mathrm{K} \alpha$ and $\mathrm{Fe} \mathrm{K} \alpha$, whose EWs exhibit a steady, monotonic rise with increasing $T$ (Papers I \& II), corresponding to a commensurate increase in the number of ionising photons. The different behaviour of $\mathrm{O} \mathrm{K} \alpha$ arises because, for coronal plasmas with $\log T \lesssim 6.6$ and solar composition, K-shell photoionisation of oxygen is due mostly to line rather than continuum radiation. Toward hotter temperatures, the important lineemitting photoionising species, including O VII and O VIII, become ionised, and continuum contributions begin to dominate.

Regarding sensitivity to $\mathrm{O}$ abundance, Figure 2 illustrates that the EW changes by a smaller factor than expected based on a proportional relation with the photospheric the $\mathrm{O}$ abundance. As discussed by Bai (1979) and in Paper I, this is a result of the the O Kshell photoionisation cross-section being a significant component of the total opacity near threshold: for very large $\mathrm{O}$ abundances where $\mathrm{O}$ begins to dominate the opacity, the EW will tend to a constant value dictated simply by equipartition of ionising photons between $\mathrm{O}$ K- and L-shells. Nevertheless, for "normal" ranges of O abundance the O K $\alpha$ line variations are significant: we find typical changes of 35-60\% over the full range of coronal temperatures for $\mathrm{O}$ abundance variations of a factor of 2 . 
When the other abundant light elements $\mathrm{C}$ and $\mathrm{N}$ are allowed to scale with the $\mathrm{O}$ abundance the diagnostic fares slightly less well but does provide discrimination between the photospheric compositions of GS and Asplund et al. (2005). The Asplund et al. (2005) O abundance is $48 \%$ lower than that of GS, and the O K $\alpha$ EWs differ by 20-25\%. For a given exciting X-ray spectrum, changes in fluorescent line strength with different photospheric parameters depend on changes in the fraction of ionising photons that ionise the parent shell of the fluorescent line in question. For a solar composition, two other sources of opacity in the vicinity of the $\mathrm{O}$ edge are $\mathrm{C}$ and $\mathrm{N}$. The chemical compositions of GS and Asplund et al. (2005) differ by $<10 \%$ in $\mathrm{O} / \mathrm{C}$ and $\mathrm{O} / \mathrm{N}$ ratios, and the lockstep changes in these elements that dilutes the effect of the different $\mathrm{O}$ abundances relative to $\mathrm{H}$ on the $\mathrm{O} \mathrm{K} \alpha \mathrm{EW}$. Despite this slightly lower sensitivity of the fluorescent line to the global chemical composition, accurate measurements of the $\mathrm{O} \mathrm{K} \alpha \mathrm{EWs}$ still potentially provide a new and relatively direct means of assessing the veracity of the GS and Asplund et al. (2005) mixtures.

The line EW is also sensitive to the abundances adopted for the exciting coronal spectrum. This is due to the large contribution of the O VII He-like complex to the source of ionising photons for coronal temperatures $\log T<6.5$. To a lesser extent, Fe L-shell and $\mathrm{Ne}, \mathrm{Mg}$ and Si H-like and He-like lines also make a contribution for temperatures up to $\log T \sim 7.0$. We have examined the sensitivity to coronal abundances by comparison of O K $\alpha$ line EWs computed for coronal spectra generated using GS and Asplund et al. (2005) compositions, as illustrated in Figure 2. The former are higher than the latter by an amount that decreases from $25-30 \%$ for temperatures $\log T \leq 6.3$ where the O VII lines dominate, to $\sim 10 \%$ at $\log T \sim 6.8-7.0$. Differences at higher temperatures are largely due to the lower $\mathrm{Ne}, \mathrm{Mg}, \mathrm{Si}$ and Fe abundances in the (Asplund et al. 2005) composition (by 74, 12, 10 and $55 \%$ respectively).

In addition to uncertainties in the solar $\mathrm{O}$ content, coronal abundance variations are also expected as a result of chemical fractionation, in which the abundances of elements with low first ionisation potentials are seen to differ from photospheric values by factors of up to $\sim 4$ (e.g. Feldman 1992). In this context, we emphasise that in a practical application of the fluorescence technique the photospheric abundance would be deduced by comparing the model fluorescent EWs computed for different photospheric abundances and the simultaneously observed coronal spectrum, and knowledge of the actual coronal composition is not required. 


\subsection{Observability of $\mathrm{O} \mathrm{K} \alpha$ Fluorescence and its Abundance Diagnostic Utility}

As discussed in Paper II in the context of Ne fluorescence, existing solar spectra generally have insufficient sensitivity to detect these relatively weak lines. While the $\mathrm{O} K \alpha$ line is expected to be an order of magnitude stronger than that of $\mathrm{Ne} \mathrm{K} \alpha$, there are essentially no high quality solar spectra that reach beyond $23 \AA$, including the most extensive sets of soft X-ray spectra obtained for the Sun by the satellite-borne Solar Maximum Mission Soft X-ray Polychromator (Acton et al. 1980) and SOLEX instruments (McKenzie et al. 1980) that had coverage up to 22.43 and $23.0 \AA$, respectively.

Existing analyses of higher quality spectra whose ranges encompassed the $\mathrm{O} \mathrm{K} \alpha$ line obtained with rocket-borne instruments (e.g. McKenzie et al. 1978; Acton et al. 1985) make no mention of a feature at the expected wavelength of $23.37 \AA$. The weakest intensity reported from the spectrum of a flare in the 10-100 $\AA$ range obtained by Acton et al. (1985) was $10 \mathrm{ph} \operatorname{arcsec}^{2}$, which can be compared with the measured O VII resonance line intensity of $508 \mathrm{ph} \mathrm{arcsec}^{2}$. At a typical solar coronal temperature of $\log T=6.3$, the flux of the O VII $\lambda 21.6$ line is a factor $\sim 10^{3}$ stronger than our prediction for the $\mathrm{O} \mathrm{K} \alpha$ line and the nondetection of the $\mathrm{O} \mathrm{K} \alpha$ line is not a surprise. While the intensity ratio drops with increasing isothermal temperature to a factor of 10 at $\log T=6.8$, where the O VII ion fraction is comparatively small, it would take the presence of relatively little cooler material within the instrument field of view to strengthen the observed O VII $\lambda 21.6$ line significantly. Accurate measurement of the $\mathrm{O} \mathrm{K} \alpha$ line requires greater sensitivity than afforded by existing solar spectra.

As shown in 93.1 , the $\mathrm{O} \mathrm{K} \alpha$ line remains essentially blend-free and isolated for plasma temperatures encountered in the non-flaring solar corona. The line EW peaks at coronal temperatures of $\log T \sim 6.2$ - conditions typical of those found in the coolest active regions and in the quiet Sun. In the case of $\mathrm{Ne} \mathrm{K} \alpha$, we found fluorescent line fluxes for quiet Sun conditions of $\sim 0.2 \mathrm{ph} \mathrm{s}^{-1} \operatorname{arcmin}^{-2}$. The $\mathrm{O} \mathrm{K} \alpha$ line is expected to be an order of magnitude stronger than this. Moreover, since the Fe XXIII and Ca XV blends remain at levels significantly below $10 \%$ of $\mathrm{O} \mathrm{K} \alpha$ for temperatures up to $\log T \sim 6.5$, the line is also expected to be accessible in much brighter active regions where fluxes are higher.

As in the case of $\mathrm{Ne} \mathrm{K} \alpha$, the large intensity contrast between $\mathrm{O} \mathrm{K} \alpha$ and neighbouring strong lines - in this case the O VII He-like complex in the range 21.6-22.1 $\AA$, and the N VII $\lambda 24.78$ resonance line -imposes tight instrumental profile requirements in order to avoid contamination of the $\mathrm{O} \mathrm{K} \alpha$ region from surrounding line wings. In the absence of suitable simultaneous spectroscopic observations of the solar regions being studied for fluorescence, such an instrument would also require fairly broad wavelength coverage so as to measure accurately the fluorescing X-ray spectrum from the $\mathrm{O} \mathrm{K}$ threshold down to wavelengths at 
which the contribution to $\mathrm{O} \mathrm{K}$ ionisation is no longer significant. The short wavelength cut-off requirement will depend on the coronal temperatures being studied.

We emphasise that in a practical application of the fluorescence technique for determining photospheric abundances, knowledge of the actual coronal composition is not required since the observed EW is compared to a measured coronal spectrum that itself must be used in model predictions.

\section{Conclusions}

We have investigated the observability of the $\mathrm{O} \mathrm{K} \alpha$ line formed in the solar upper photosphere/lower chromosphere by fluorescence from overlying coronal X-radiation. While quite faint compared to the prominent lines of abundant coronal ions, $\mathrm{O} \mathrm{K} \alpha$ should be essentially unblended at plasma temperatures up to $\log T \sim 6.5$.

The line EW peaks at temperatures $\log T \sim 6.2$ with a value of $\sim 0.1 \AA$, and we estimate an $\mathrm{O} \mathrm{K} \alpha$ flux of $\sim 2 \mathrm{ph} \mathrm{s}^{-1} \operatorname{arcmin}^{-2}$ for the quiet Sun at heliocentric angles close to $0^{\circ}$ - an order of magnitude larger than predicted in Paper II for Ne K $\alpha$ fluorescence. Both lines should be quite observable with instruments adequately tailored toward high resolution $\lambda / \Delta \lambda \gtrsim 1000$ and sufficiently well-behaved instrumental profile wings.

The $\mathrm{O} \mathrm{K} \alpha$ line provides a potentially accurate absolute $\mathrm{O}$ abundance diagnostic, provided abundances of other prominent light elements are also constrained. It should also be possible to distinguish between the currently competing photospheric compositions of GS and Asplund et al. (2005), provided line EWs can be determined to an accuracy significantly better than 20\%. We conclude that X-ray fluorescence of O (and Ne) represents a viable means for resolving the current solar abundance-helioseismology conundrum.

We thank the NASA AISRP for providing financial assistance for the development of the PINTofALE package. JJD was funded by NASA contract NAS8-39073 to the Chandra $X$-ray Center during the course of this research and thanks the Director, H. Tananbaum, for continuing support and encouragement. BE was supported by Chandra Grants GO67008X and GO6-7098X. Finally, we thank the anonymous referee for comments that helped us clarify the manuscript. 


\section{REFERENCES}

Acton, L. W., Bruner, M. E., Brown, W. A., Fawcett, B. C., Schweizer, W., \& Speer, R. J. 1985, ApJ, 291, 865

Acton, L. W., Finch, M. L., Gilbreth, C. W., Culhane, J. L., Bentley, R. D., Bowles, J. A., Guttridge, P., Gabriel, A. H., Firth, J. G., \& Hayes, R. W. 1980, Sol. Phys., 65, 53

Antia, H. M. \& Basu, S. 2005, ApJ, 620, L129

Asplund, M., Grevesse, N., \& Sauval, A. J. 2005, in ASP Conf. Ser. 336: Cosmic Abundances as Records of Stellar Evolution and Nucleosynthesis, 25

Ayres, T. R., Plymate, C., \& Keller, C. U. 2006, ApJS, 165, 618

Bahcall, J. N., Basu, S., Pinsonneault, M., \& Serenelli, A. M. 2005a, ApJ, 618, 1049

Bahcall, J. N., Basu, S., \& Serenelli, A. M. 2005b, ApJ, 631, 1281

Bai, T. 1979, Sol. Phys., 62, 113

Basko, M. M. 1978, ApJ, 223, 268

Basu, S. \& Antia, H. M. 2004, ApJ, 606, L85

Caldwell, C. D., Schaphorst, S. J., Krause, M. O., \& Jimnez-Mier, J. 1994, J. Electron. Spectrosc. Relat. Phenom., 67, 243

Delahaye, F. \& Pinsonneault, M. H. 2006, ApJ, 649, 529

Drake, J. J. \& Ercolano, B. 2007, ApJ, 665, L175

Drake, J. J., Ercolano, B., \& Swartz, D. 2007, ApJ submitted

Drake, J. J. \& Testa, P. 2005, Nature, 436, 525

Ercolano, B., Barlow, M. J., \& Storey, P. J. 2005, MNRAS, 362, 1038

Ercolano, B., Barlow, M. J., Storey, P. J., Liu, X.-W., Rauch, T., \& Werner, K. 2003, MNRAS, 344, 1145

Feldman, U. 1992, Physica Scripta Volume T, 46, 202

García, J., Mendoza, C., Bautista, M. A., Gorczyca, T. W., Kallman, T. R., \& Palmeri, P. 2005, ApJS, 158, 68 
Grevesse, N. \& Sauval, A. J. 1998, Space Science Reviews, 85, 161

Juett, A. M., Schulz, N. S., \& Chakrabarty, D. 2004, ApJ, 612, 308

Kashyap, V. \& Drake, J. J. 2000, Bulletin of the Astronomical Society of India, 28, 475

Krause, M. O. 1979, Journal of Physical and Chemical Reference Data, 8, 307

—. 1994, Nuclear Instruments and Methods in Physics Research B, 87, 178

Landi, E., Del Zanna, G., Young, P. R., Dere, K. P., Mason, H. E., \& Landini, M. 2006, ApJS, 162, 261

Landi, E., Feldman, U., \& Doschek, G. A. 2007, ApJ, 659, 743

Lin, C.-H., Antia, H. M., \& Basu, S. 2007, ArXiv e-prints, 706

Mazzotta, P., Mazzitelli, G., Colafrancesco, S., \& Vittorio, N. 1998, A\&AS, 133, 403

McKenzie, D. L., Landecker, P. B., Broussard, R. M., Rugge, H. R., Young, R. M., Feldman, U., \& Doschek, G. A. 1980, ApJ, 241, 409

McKenzie, D. L., Rugge, H. R., Underwood, J. H., \& Young, R. M. 1978, ApJ, 221, 342

Parmar, A. N., Culhane, J. L., Rapley, C. G., Wolfson, C. J., Acton, L. W., Phillips, K. J. H., \& Dennis, B. R. 1984, ApJ, 279, 866

Ralchenko, Y., Jou, F.-C., Kelleher, D. E., Kramida, A. E., Musgrove, A., Reader, J., Wiese, W. L., \& Olsen, K. 2007, in NIST Atomic Spectra Database (version 3.1.2), National Institute of Standards and Technology, Gaithersburg, MD, http://physics.nist.gov/asd3

Schmelz, J. T., Nasraoui, K., Richardson, V. L., Hubbard, P. J., Nevels, C. R., \& Beene, J. E. 2005, ApJ, 627, L81

Socas-Navarro, H. \& Norton, A. A. 2007, ApJ, 660, L153

Stolte, W. C., Lu, Y., Samson, J. A. R., Hemmers, O., Hansen, D. L., Whitfield, S. B., Wang, H., Glans, P., \& Lindle, D. W. 1997, Journal of Physics B Atomic Molecular Physics, 30, 4489

Turck-Chièze, S., Couvidat, S., Piau, L., Ferguson, J., Lambert, P., Ballot, J., García, R. A., \& Nghiem, P. 2004, Physical Review Letters, 93, 211102

Vernazza, J. E., Avrett, E. H., \& Loeser, R. 1981, ApJS, 45, 635 
Zaatri, A., Provost, J., Berthomieu, G., Morel, P., \& Corbard, T. 2007, A\&A, 469, 1145

This preprint was prepared with the AAS LATEX macros v5.2. 
Table 1: Lines in the CHIANTI 5 database lying within $\pm 0.07 \AA$ ( $5 \sigma+$ a rest wavelength uncertainty $0.02 \AA$ ) of the position of the $23.37 \AA$ O K $\alpha$ transition for a Gaussian resolving power of $\lambda / \Delta \lambda=1000$. Only lines with predicted intensities $\geq 1 \times 10^{-4}$ that of the brightest line are listed.

\begin{tabular}{lcccc}
\hline Ion & $\lambda(\AA)$ & Rel. Int. & $\log T_{\max }$ & Transition \\
\hline Fe XXIII & 23.303 & 0.54 & 7.2 & $2 s 3 p^{3} P_{2}-2 s 5 d^{3} D_{3}$ \\
Ca XV & 23.305 & 0.07 & 6.6 & $2 s^{2} 2 p^{2}{ }^{1} D_{2}-2 s^{2} 2 p^{3} d^{3} D_{1}$ \\
Ca XVI & 23.320 & 0.03 & 6.7 & $2 s 2 p^{2}{ }^{2} P_{3 / 2}-2 s 2 p\left({ }^{3} P\right) 3 d^{4} D_{5 / 2}$ \\
Fe XXIII & 23.321 & 0.10 & 7.2 & $2 s 3 p^{3} P_{2}-2 s 5 d^{3} D_{2}$ \\
Ca XVI & 23.341 & $1 \times 10^{-3}$ & 6.7 & $2 s 2 p^{2}{ }^{2} P_{3 / 2}-2 s 2 p\left({ }^{3} P\right) 3 d^{4} D_{3 / 2}$ \\
Ar XVI & 23.343 & 0.01 & 6.7 & $1 s^{2} 2 s^{2} S_{1 / 2}-1 s^{2} 3 d^{2} D_{5 / 2}$ \\
Ar XVI & 23.362 & 0.01 & 6.7 & $1 s^{2} 2 s^{2} S_{1 / 2}-1 s^{2} 3 d^{2} D_{3 / 2}$ \\
Fe XXIII & 23.363 & 1.00 & 7.2 & $2 s 3 p^{3} P_{1}-2 s 5 s{ }^{1} S_{0}$ \\
Fe XXIII & 23.381 & 0.04 & 7.2 & $2 s 3 p{ }^{1} P_{1}-2 s 5 d^{3} D_{1}$ \\
Fe XXIII & 23.388 & $2 \times 10^{-4}$ & 7.2 & $2 s 3 p^{1} P_{1}-2 s 5 p^{3} P_{1}$ \\
Fe XXIII & 23.388 & $3 \times 10^{-4}$ & 7.2 & $2 s 3 p^{1} P_{1}-2 s 5 p{ }^{1} P_{1}$ \\
Ca XV & 23.388 & 0.88 & 6.6 & $2 s^{2} 2 p^{2}{ }^{1} S_{0}-2 s^{2} 2 p^{3} d{ }^{1} P_{1}$ \\
Fe XXIII & 23.406 & $8 \times 10^{-3}$ & 7.2 & $2 s 3 p^{3} P_{2}-2 s 5 d^{3} D_{1}$ \\
Fe XXIII & 23.412 & $3 \times 10^{-4}$ & 7.2 & $2 s 3 p^{3} P_{2}-2 s 5 p^{3} P_{1}$ \\
Ca XV & 23.415 & 0.45 & 6.6 & $2 s^{2} 2 p^{2}{ }^{1} D_{2}-2 s^{2} 2 p^{3} d^{3} F_{3}$ \\
Ni XXVIII & 23.439 & $5 \times 10^{-4}$ & 8.0 & $3 p{ }^{2} P_{1 / 2}-4 d^{2} D_{3 / 2}$ \\
\hline
\end{tabular}




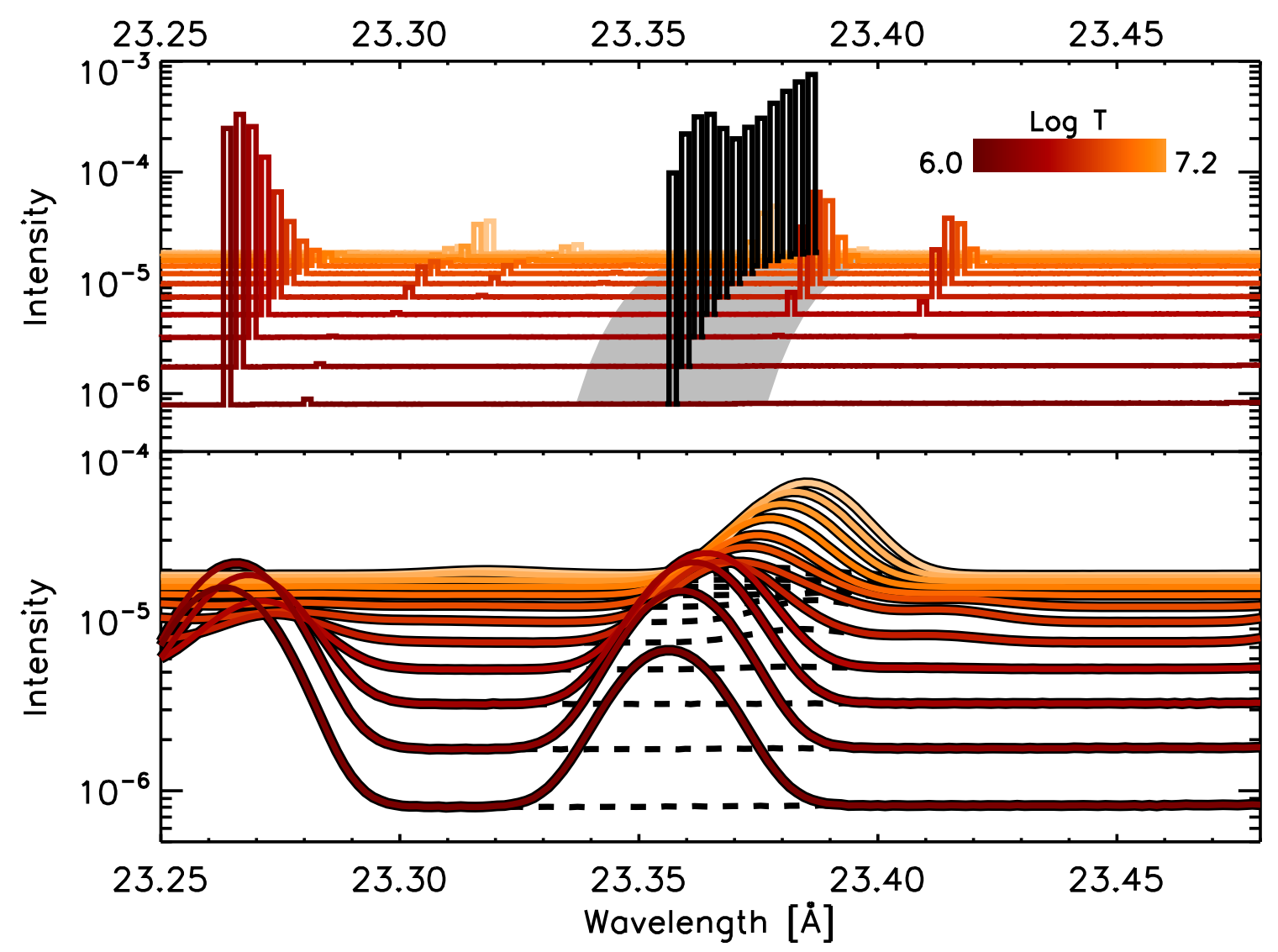

Fig. 1.- Top: The strength of the $\mathrm{O} \mathrm{K} \alpha$ shown in comparison to neighbouring and blending lines in the fluorescing coronal X-ray spectrum for coronal isothermal plasma temperatures in the range $10^{6}-10^{7.2} \mathrm{~K}$ (wavelengths shown off-set for clarity). The underlying grey shaded region represents the uncertainty in the wavelength of the $\mathrm{O} \mathrm{K} \alpha$ line. Bottom: The same coronal spectra with (solid curves) and without (dashed) the addition of $\mathrm{O} \mathrm{K} \alpha$ smoothed to a resolving power (FWHM) of $\lambda / \Delta \lambda=1000$. 


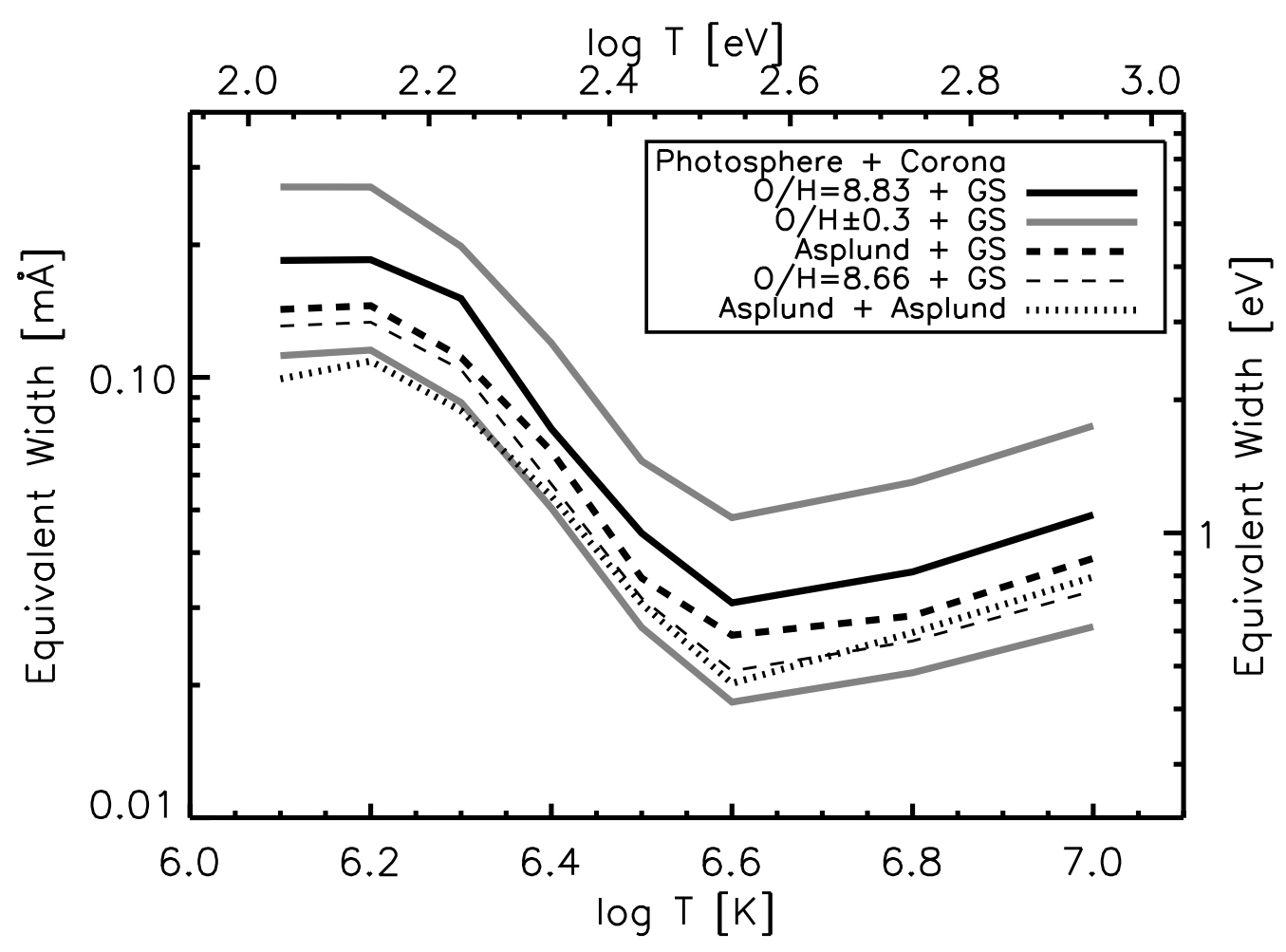

Fig. 2. - The equivalent width of the $\mathrm{O} \mathrm{K} \alpha$ line with respect to the ionising coronal $\mathrm{X}$ ray spectrum for heliocentric angle $\theta=0$ as a function of isothermal plasma temperature, computed for different coronal and photospheric chemical compositions: GS corona + GS photosphere (solid black curve), GS corona + GS photosphere with the O abundance differing by factors of 2 (solid grey), GS corona + Asplund et al. (2005) photosphere (heavy dashed), GS corona + GS photosphere with Asplund et al. (2005) $\mathrm{O}$ abundance $(\mathrm{O} / \mathrm{H}=8.66$; light dashed); and Asplund et al. (2005) corona + Asplund et al. (2005) photosphere (black dotted). 